\title{
El duelo: que modelización?
}

\author{
Flora Singer
}

El tema del duelo no ha sido tratado en psicoanálisis en forma acorde a su importancia psicopatológica. Hay obstáculos epistemológicos para ello, que tienen que ver con el tratamiento del pasaje del objeto real del duelo a la interioridad de su pathos. La autora considera que una lógica paradojal y de lo negativo permite articular ese pasaje: contemplar la diferen-ciación entre realidad externa-realidad interna, pero también integrar la realidad externa en el análisis. Lo negativo está constituído, entre otros aspectos, por la compleja zona de intercambios entre la realidad externa del duelo y la realidad interna.

El modelo del duelo es un ejemplo de la importancia metodológica de una lógica pa-radojal y de lo negativo, que permite una permeabilidad en los límites de los modelos, por la cual se mantienen a la vez la especificidad del modelo y la integración de una alteridad, en este caso, la realidad del objeto del duelo. 


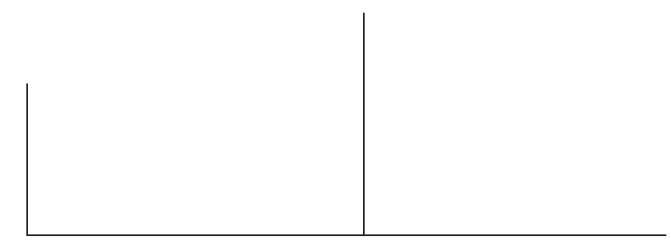

El tema del duelo no ha sido en psicoanálisis tratado en forma proporcional a su importancia psicopatológica, como si este tema circulara de alguna manera en los límites del psicoanálisis. Hay; a nuestro entender, razones históricas para ello, a partir del campo circunscripto por Freud para abordarlo, y razones del orden de un obstáculo epistemológico. Nuestra hipótesis es que el tema del duelo toca los límites del psicoanálisis, más aún, permite interrogar esos límites y la cualidad de la que están constituídos. La confrontación de dos modelos en relación al duelo, el modelo freudiano y el de los desarrollos recientes de Jean Allouch en torno al tema, nos permitirán una reflexión que se extiende a toda modelización en psicoanálisis, su lógica interna, coherente con una lógica de la paradoja y lo negativo, y la cualidad de sus límites.

Desde las primeras líneas de "Duelo y melancolía", Freud manifiesta su intención de "aclarar la esencia de la melancolía comparándola con el afecto normal del duelo", así como ya se había servido del modelo normal del sueño para desarrollar las alteraciones narcisistas. Aún cuando en este texto el tema principal sea la melancolía, el esbozo que Freud hace del duelo ha jugado un rol paradigmático para la concepción del duelo en psicoanálisis.

Freud define el duelo como "la reacción frente a la pérdida de una persona amada o de una abstracción que haga sus veces, como la patria, la libertad, un ideal etc.". En la medida en que se espera que el duelo será superado naturalmente luego de un cierto tiempo, ello lleva a Freud a afirmar que no se trata de un estado patológico, y que hasta sería inoportuna toda intervención en el mismo. 


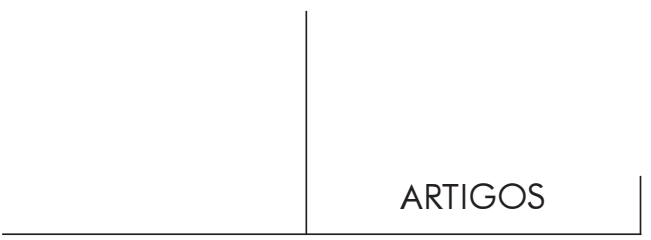

El duelo severo comporta, como la melancolía, la pérdida de interés por el mundo exterior, en la medida en que este último no recuerda al difunto.

En la melancolía, la pérdida es conocida del enfermo, pero no así lo que éste ha perdido en esa persona; en el duelo por el contrario, nada de lo que concierne a esa persona es inconsciente. En el caso de la melancolía no se puede saber lo que absorbe tan completamente al sujeto: hay un trabajo interior desconocido. En el duelo, el trabajo que absorbe al yo explica su ausencia de interés en relación al mundo exterior. La libido permanece ligada al objeto perdido en cada uno de sus recuerdos, hasta que la realidad se impone, el yo termina por aceptar que el objeto no existe más, y escoge permanecer vivo y romper su lazo con el objeto muerto. Esta ruptura es lenta y progresiva, y el duelo concluye cuando el yo puede renunciar al objeto declarándolo muerto.

J. Allouch analiza los límites de la concepción freudiana del duelo. La caracterización del duelo como fenómeno normal tuvo como consecuencia por un lado, borrar la especificidad del duelo, y por otro, la de introducir una normatividad en el análisis. Allouch dice que allí donde, a partir de algo conocido - el duelo - se iría a explicar lo desconocido - la melancolía -, se terminó tomando por conocido lo que de hecho se conocía mal, y una versión del duelo se filtra así subrepticiamente. Las consecuencias de ese modelo del duelo se hacen aún sentir en una cierta ortodoxia freudiana.

Ese modelo del duelo se apoya sobre un trabajo de duelo que apunta a desinvestir el objeto muerto, a liberar la libido, y finalmente a emplear esta última en investir un objeto sustitutivo. Las nociones claves de "trabajo de duelo", "objeto sustitutivo" y "prueba de realidad" no han sido suficientemente desarrolladas, y sientan una normativa que oscurece más de lo que aclara el tema.

A partir de una crítica del modelo freudiano, Allouch desarrolla otro modelo, basado en una serie de parámetros tomados de la teoría lacaniana.

Para Allouch el duelo es patología, y no normalidad. Al mismo tiempo, estima que una clínica del duelo apunta más al reconocimiento de una pluralidad de duelos y a su red diferencial, que a la búsqueda de una normativa generalizadora.

Allouch señala que para Freud el duelo es una operación que no deja resto, mientras que para Lacan, hay disparidad entre la situación anterior y la ulterior al duelo, y lo que así se inscribe, es la esencial no sustitución del objeto.

El duelo no es la separación con el muerto, es un cambio en la relación al muerto. Se trata de una alteración en la relación de objeto, y la producción de una nueva figura de la relación de objeto.

Las identificaciones a los rasgos del objeto muerto tampoco tienen como función la separación con el objeto, sino por el contrario, apuntan a mantener la relación con dicho objeto. 


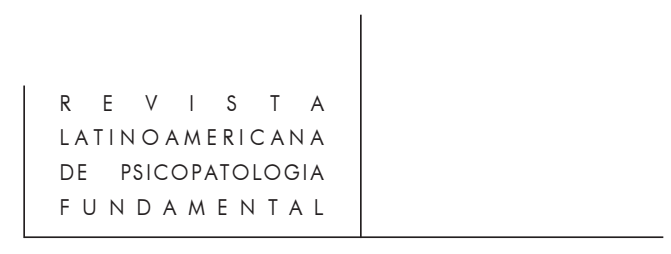

Todo ello lleva a la instauración de una nueva posición subjetiva. Aquel que está de duelo es habitado por el ser que ha perdido. La posición del sujeto frente a su muerto interviene en las instancias más cruciales de su vida, en las más anodinas, en sus síntomas: compromete todo su ser.

El objeto de deseo es un objeto del cual se ha perdido la correspondencia; el objeto del duelo mantiene una correspondencia con la realidad demasiado densa. Hay un "agujero en lo real" (de acuerdo a J. Lacan) que hace que el duelo se constituya como para-psicótico.

El duelo pasa de la experiencia de desaparición del ser querido y del déficit de la realidad para resolver esta cuestión, al reconocimiento de su inexistencia. Ello no puede ser admitido sino al final del duelo.

La efectuación del duelo pasa por el sacrificio de una parte de sí. "La muerte convoca la muerte". Allouch sostiene que aquel que está de duelo, sigue al muerto a su tumba, ofreciendo así un trozo de sí.

No hay duelo que transcurra exclusivamente en lo simbólico, sino que el duelo compromete la totalidad del sujeto.

Finalmente, Allouch señala otro elemento: la medida del horror de aquel que está de duelo, guarda una relación con la no-realización en la vida del muerto. Aquello que la muerte vuelve definitivamente no realizado en el muerto, deja también su traza en aquel que está de duelo.

En este breve planteo de los dos modelos del duelo, los límites del desarrollo freudiano se hacen más manifiestos. Que el desarrollo freudiano en torno al duelo sea insuficiente es comprensible, en la medida en que Freud apunta al desarrollo de la melancolía. La falta de desarrollo en sí no constituiría un problema, en la medida en que dicho tema pudiera continuar siendo desarrollado metapsicológicamente a partir de sus esbozos, como ha sido el caso de otros temas.

Hay en la manera en que Freud ubica el tema del duelo, obstáculos metodológicos que impiden su ulterior desarrollo metapsicológico, como si el duelo transcurriera en los límites del psicoanálisis. Freud, que pudo abrir un camino para su teoría más allá de los recursos que su tiempo le proveía, se detuvo ante el duelo cuya "normalidad" se le impuso como un límite al campo psicoanalítico.

Freud coloca de entrada el duelo en el terreno de la realidad y el de la conciencia, y ello da lugar a numerosos deslizamientos positivizantes, lo que vuelve difícil su integración metapsicológica.

En relación al trauma y al fantasma, Freud pudo instaurar una dialéctica tal que le permitió reinstaurar el trauma en el psiquismo. Los desarrollos postfreudianos pudieron en ese terreno, salvar las aporías que pueden originarse en la distinción realidad interna-realidad externa, y continuar desarrollando el tema. El objeto originario es un objeto doblemente perdido: perdido en el psiquismo, y, 


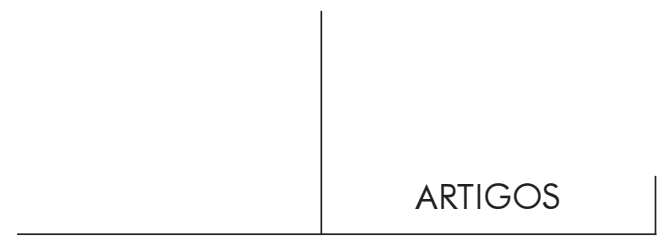

como dice Allouch, un objeto del cual se ha perdido también la correspondencia. El objeto del duelo, es un objeto real, del que lo que cambia en relación a su correspondencia interna, es su propia realidad.

La realidad externa vuelve confuso el análisis en torno al duelo, y no es fácil de ubicar. Freud la ubica en un circuito externo al psicoanálisis, en la línea conciencia-elaboración, y establece así una correspondencia entre realidad externa y positividad. Este ordenamiento se hace presente ya en las primeras líneas de "Duelo y melancolía": "El duelo es, por regla general, la reacción a la pérdida de una persona amada o de una abstracción que haga sus veces, como la patria, la libertad, un ideal etc."

Esta manera de abordar la cuestión del duelo, pone el acento más sobre la vertiente psíquica que sobre la cualidad del objeto externo, ya que poco importa que éste sea persona amada o abstracción. Esta falta de integración entre realidad interna-realidad externa, tuvo consecuencias bastante considerables en el postfreudismo.

La cualidad del objeto real tiene destinos internos diferentes, pero ello no ha sido tomado en cuenta en "Duelo y melancolía". Falta sobre todo el pasaje de una exterioridad particular a una interioridad. Hay una diferencia significativa entre la pérdida de una persona amada y la de una abstracción, así como entre un ser amado muerto y un novio abandonante, a menos que se subordine toda categorización de los objetos a la jerarquía de los objetos internos, y que se tome éstos como específicos al campo psicoanalítico, excluyendo los otros. Este clivaje deriva de una metodología que subrepticiamente establece límites bien definidos entre el afuera y el adentro, borrando así las différances que permiten el pasaje. También las différances entre duelo-separación-pérdida quedan así borradas.

La prioridad dada a la realidad psíquica como territorio interno al psicoanálisis, no le quita sin embargo nada a la necesidad de apelar a la realidad externa. El territorio psicoanalítico está delimitado por objetos internos, pero sus avatares están asimismo ligados a su proveniencia. Uno de los factores que ha impedido promover el desarrollo de la temática del duelo en psicoanálisis ha sin duda sido del orden de este tipo de dificultades metodológicas. El desarrollo del tema del duelo ha sido hecho históricamente sobre la base de equivalencias demasiado rápidas a otras categorías - como la de pérdida, por ejemplo, que tiene otra connotación -, lo que ha hecho obstáculo a una posible clínica diferencial del duelo.

Los límites y consecuencias de esta metodología vuelven manifiesta la necesidad de instaurar el negativo de una zona-entre. Una lógica paradojal y el reconocimiento de la heterogeneidad de los términos en el pasaje, preserva la especificidad de éstos y permite en este caso, la integración de la realidad externa en el análisis. 


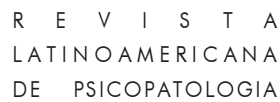

Hemos desarrollado en otros trabajos cómo, por las características de su objeto, el psicoanálisis aporta una experiencia de alteridad. La alteridad del inconsciente es una alteridad particular: el inconsciente es un otro negativo, que no se presenta como pleno a nivel del logos. Ello determina una epistemología específica, constituída por una lógica de la paradoja, que a diferencia de la lógica de la identidad, permite la inclusión de lo otro, en una relación dinámica entre lo uno y lo otro. Ambos conservan su especificidad, pero también se establece entre ambos una zona-entre de transformaciones.

Denominamos negativo al enigma, al límite al saber, en dos aspectos:

1) lo anterior a una posible elaboración, y

2) las zonas-entre de pasaje entre lo uno y lo otro, zonas de transformaciones en donde los límites entre ambos términos plantean igualmente un límite al saber: son indecidibles.

Los modelos psicoanalíticos, en forma coherente con lo anterior, guardan una permeabilidad en sus límites, por la cual se mantienen a la vez la especificidad del modelo y el pasaje de lo otro. Son en particular las fronteras del psicoanálisis, desde la clínica hasta la teoría, que interpelan así sus modelos. El modelo del duelo, en la medida en que debe integrar la realidad externa de la muerte, puede constituirse como un ejemplo de la importancia metodológica del reconocimiento de una lógica paradojal y de lo negativo. Su utilidad se torna manifiesta en la temática del duelo, ya que una de las dificultades de esta última es la de ser abordada desde un pensamiento positivizado, insuficiente para dar cuenta de la complejidad del proceso.

El forcing en el tratamiento de la realidad lleva a Freud a hacer una distinción entre la realidad de la pérdida en el caso del duelo, y la naturaleza del objeto de la melancolía. Aún cuando la melancolía es provocada por una pérdida real, el sujeto está en medida de saber a quién ha perdido, pero no aquello que ha perdido en esa persona. Esta distinción, que tiene un valor heurístico del lado de la melancolía, conduce a un cierre del lado del duelo, puesto que mientras en la melancolía la pérdida del objeto se sustrae a la consciencia, y hace la impresión de un enigma, en el caso del duelo "no hay nada inconciente en lo que atañe a la pérdida". Con lo cual, el trabajo del duelo se considera un trabajo razonable, a cuenta de la aceptación del principio de realidad.

La positividad de la realidad se desliza sobre la expresión de "trabajo de duelo", que se toma entonces en el sentido de una elaboración que permite superar la pérdida. Se trata de un trabajo tomado en el sentido de un progreso hacia un fin. El fin borra el proceso mismo. El fin del trabajo cuenta más que los obstáculos que se interponen para su cumplimiento (obstáculos en donde residiría el trabajo propiamente analítico, que queda así elidido). La nominación misma del duelo se pervierte en ese finalismo, puesto que lo que en ese caso se nomina duelo es un 


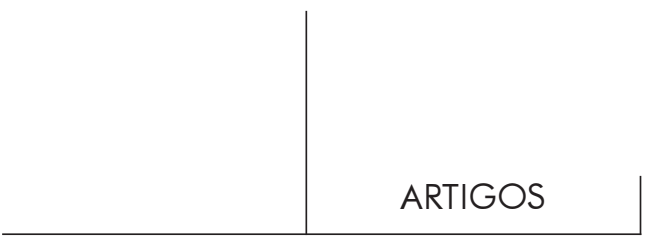

duelo logrado, superado. De otro modo, se trata de un duelo patológico o melancólico. La normatividad toma así la delantera en relación a la clínica.

La homología de lo normal con lo real ha estatuído una normativa que le ha quitado al duelo su estatuto psicopatológico. Lo psicopatológico no es lo contrario a lo normal, sino lo que conlleva un pathos, en el sentido de pasión, sufrimiento. Entre lo normal y lo patológico también se estatuye así, un espacio de intercambios.

Los pilares que sustentan la concepción freudiana del duelo, lo haya Freud querido o no, dejan inanalizado el proceso - el trabajo - del duelo por el cual el objeto es dado finalmente como perdido, lo que de hecho constituye el fin del proceso complejo del duelo.

Del lado de la cualidad de la pérdida, y del lado de lo que ha sido perdido en la realidad, estos dos aspectos hacen a la especificidad de la clínica del duelo. Entre el objeto real y el objeto perdido, ese proceso que constituye el duelo, comporta una serie de instancias. El final del proceso de duelo comporta la aceptación de esta transformación del objeto real en objeto perdido, y el proceso de duelo tiene que ver con la especificidad de esas dos cualidades del objeto: la especificidad del objeto real muerto, y la del objeto perdido del duelo, que no es el mismo objeto que el objeto perdido interno, objeto de deseo, aún sin hay cruzamientos que ponen en relación uno con otro. El objeto no puede no ser tomado en cuenta: ni adquirir una realización positivizante que lo hace salir fuera del campo psicoanalítico, ni ser equiparado al fantasma.

A. Green llama función objetalizadora aquella que, por actividad de enlace, crea nuevos objetos, lo que torna complejo el campo objetal ya que, en lugar de circunscribir objetos precisos, permite colocar el acento en los procesos de producción y las relaciones entre los objetos. Más que un realismo de los objetos y los impases producidos por sus falsas oposiciones, se puede de esa manera someter al análisis un haz dinámico objetal.

En el duelo, lo que primero se impone al psiquismo proviene de la realidad, y como dato primero, no se trata de una ausencia. Si hay trabajo elaborativo, es a tener en cuenta ese decalaje en relación a esta imposición de la realidad, que sobreviene en primer lugar. La inscripción de la ausencia sobreviene seguidamente a una elaboración. Lo que se impone primero es una realidad otra, por la cual aquel que ayer estaba, no lo está hoy.

El golpe que la realidad impone es el de una realidad otra, una realidad tan plena, que hasta es plena en demasía. Lo negativo en su primera acepción está constituído por aquello que se impone así, antes de toda elaboración. No es el vacío, la ausencia, sino una alteridad en el seno de la realidad la que debe ser integrada. El muerto no es un ausente, al menos como punto de partida. Lo unheimlich del muerto, ausente pero muy presente, se impone antes que nada 


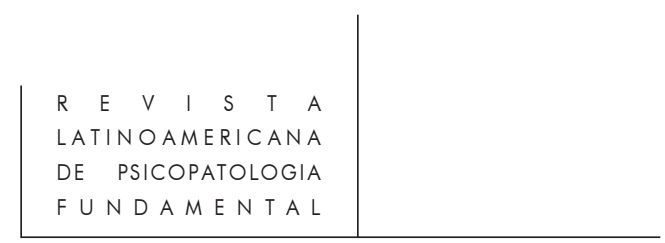

como alteridad. Esta primera acepción de lo negativo es la de un otro a ser integrado.

El trabajo de duelo cae en la segunda acepción de lo negativo: es un espacioentre, que hace de enlace entre el sujeto viviente y el muerto, lo real y la elaboración. Ese trabajo no es equivalente a una simbolización, aunque ésta pueda integrarlo. Se trata de un espacio-entre productivo, en donde se produce la mezcla: mezcla del sujeto en vida y muerto, de lo simbólico y lo real, del síntoma y el delirio. La dialéctica de la desinvestidura del muerto y la investidura de un objeto sustitutivo despierta un imaginario circunscrito y definido de los términos. Por el contrario, el proceso de duelo tiene que ver con las différances: el sujeto en vida es desinvestido y reinvestido como muerto. En el espacio-entre que para ello se instaura, si el muerto es dado finalmente como ausente, ello lo es a cuenta de un reacomodamiento psíquico, al cabo del cual continuará estando investido, pero en un lugar otro.

En el duelo hay un proceso de investidura que va del objeto muertonegativizado al objeto muerto-ausente. Ello conlleva una paradoja que torna intrincada la relación entre pulsión de vida y pulsión de muerte, ya que lo que así es investido, es la muerte. "La muerte llama a la muerte", dice Allouch.

La intrincación pulsion de vida/pulsión de muerte no se resume en un conflicto ambivalencial, si bien éste puede integrarla. Cuando la muerte es investida de esta manera, la aproximación a lo negativo de la muerte puede asumir una diversidad de formas en donde el pathos, el sufrimiento, deja su traza. El pathos de la enfermedad, del síntoma, del delirio. Se puede matar al muerto, forma del sadismo. Se puede identificarse con el muerto: al muerto ya muerto, al muerto cuando estaba aún en vida, a los aspectos muertos del mismo en vida. La importancia acordada por Allouch a los aspectos irrealizados del muerto juegan también como otras tantas aproximaciones a un espacio de intercambios que se instaura en ocasión del duelo, espacio-entre la vida y la muerte.

El "pequeño sacrificio de sî́" que señala Allouch, es la traza de la muerte en el sujeto vivo. Ese pedazo, ¿a quién pertenece? se pregunta Allouch. De hecho, pertenece tanto al muerto como al vivo. Objeto investido que representa al muerto, pero también objeto narcisista que concentra la parte muerta del que queda en vida, que de esa manera acompaña al muerto muriendo con él. De la misma forma que había identificaciones a la persona en vida, hay nuevas identificaciones con el muerto en tanto muerto, lo que arrastra una compleja dialéctica entre libido de objeto y libido narcisista.

Finalmente, el pathos del duelo provoca un reacomodamiento del psiquismo. En la elaboración del duelo, el retorno no es a cero, como lo manifiesta Lacan. La elaboración no está tampoco, constituída exclusivamente por lo simbólico. 


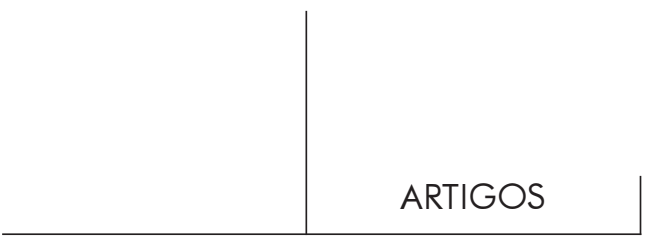

Un fragmento de análisis citado por F. Gantheret con otros fines en su trabajo "Per via di levare", sirve para ilustrar la complejidad del proceso de duelo:

...la madre finalmente murió... Ella estaba sola, y se sintió acosada por el empleado de las pompas fúnebres que tenía papeles a hacerle llenar, antes de poder acceder al cuarto donde se realizaba el velatorio... ella se aproximó, y contempló el rostro y las manos de su madre. Un ligero sobresalto se apoderó de su espíritu, tuvo el impulso de besarla, luego dudó. Ya cerraban el féretro. Pero entonces... ella no había visto la piel de su madre fresca y - si la hubiera tocado - seguramente tibia? Acaso ella no había visto levantarse su pecho ligeramente como si - sí! aún respirara?... Tomó su lugar en el furgón presa de terribles tormentos. Se reprochaba no haber intervenido más firmemente, de haber cedido. El horror se apoderó aún de ella cuando descendieron el féretro a la fosa. Ella hubiera querido gritar: "Deténganse... yo creo... quizás..." ...

A las once todo había terminado y se encontró sola en el cementerio.

Allí permaneció toda la jornada, debió salir a la noche, pero permaneció dando vueltas en las cercanías... A la mañana entró en una comisaría y dijo...: "Por favor, se podría abrir la tumbra de la Sra. X? es mi madre, y creo... quizás aún respira”.

Así fue conducida al hospital psiquiátrico, y es entonces que la ví por primera vez. Sólo tenía treinta y siete años, pero parecía muchos más; en realidad no tenía edad, grande, vestida de negro, corpulenta, informe. Manifestaba gran cortesía, pero se la experimentaba enteramente en su delirio...

Trabajé mucho tiempo y bien, según creo, con ella. Le hice interpretaciones juiciosas y obtuve resultados consecuentes. El delirio desapareció poco a poco...

Los recuerdos se orientaban cada vez más hacia su infancia: feliz, plena de juegos y de paisajes. Poco a poco la imagen de la madre se relativizó: Jeanne comenzó a experimentar hacia ella sentimientos hostiles. Volvió a recordar su casamiento: quizás ese fracaso fuera debido en parte a su madre, que no le había advertido nada sobre las cosas del sexo? A su madre tampoco le gustaba ese casamiento: temía ser dejada de lado. Las exigencias de sus enfermedades le habían impedido salir, "frecuentar". Se remontó a su infancia: a veces el padre la llevaba a pasear, cuando ella estaba de vacaciones; de pequeña, tenía un pequeño asiento para ella en su bicicleta. Más grande, ella tuvo una propia.

Recuerdos soleados de las rutas de la campiña con su padre... A la madre no le gustaban esas salidas de a dos y se oponía a ellas con frecuencia. Eso "fatigaba a la pequeña". Ya entonces...

Así se descubría un paisaje edípico. El delirio desapareció. O más bien, dejó lugar a otro proceso: Jeanne se tornó hipocondríaca. El duelo se jugaba ahora en torno a objetos parciales tomados del cuerpo de la madre, introyectados y atormentadores en el interior de su propio cuerpo. Continué el trabajo a ese nivel. Pudimos establecer relaciones entre lo que ella decía de su madre y sus "piernas malas" o sus problemas intestinales...

La hipocondría remitió. Ella pudo abandonar el hospital, y vino a verme en consulta, primero todas las semanas, luego cada quince días... Aparentemente todo 
transcurría bien. Había obtenido un apartamento de dos piezas, cerca de su trabajo.

Nuestras entrevistas se fueron espaciando, y luego se interrumpieron. Un día le pedí a la asistente social del servicio... que pasara a ver a la Sra. Jeanne.

La encontró en su apartamento, en el mismo lugar. La Sra. Jeanne había recuperado los muebles que habían sido guardados en un depósito después de la quiebra del almacén: "los muebles de mis padres". Invadían todo el espacio de su apartamento: apenas era posible deslizarse a lo largo de los muros. El cuerpo de la Sra. Jeanne rehabitado por los pedazos de su madre. El cuerpo de su madre restaurado. El espacio del amor (no la escena primitiva, el amor) recuperado, y Jeanne vuelta niña en su mundo restaurado. En medio de ello, conservando su trabajo, sola, la Sra. Jeanne, a los cuarenta años, envejecía dignamente, un poco original, es verdad, pero...

El recorrido del "trabajo de duelo" es el de un pathos - el duelo no puede sino ser psicopatológico - que lleva a un reacomodamiento psíquico. Se introducen así nuevas investiduras que llevan la marca de lo negativo, ese espacioentre signado por los muebles, objetos del amor, objetos de la muerte. La muerte de los otros, y la de una parte de sí mismo. Objetos que simbolizan los aspectos vivos del pasado, pero también los aspectos inertes del presente. Investiduras, sí, pero también investiduras de la muerte. Producción en torno a lo negativo de la muerte con recursos simbólicos, pero también con mecanismos restitutivos: delirio, síntomas. El psiquismo pone así en marcha un trabajo - en el sentido de una producción - en torno a lo negativo. El trabajo de duelo está constituído por ese trabajo complejo, y no sólo por una elaboración positiva que apunta a colmar el agujero de la pérdida. Es alrededor de un agujero que se produce la elaboración. Qué queda del agujero y qué de la elaboración tiene menos que ver con una economía sustitutiva que con una estructuración compleja al final de la cual hay cambio y permanencia de todos los términos.

Lo negativo en el proceso de duelo está connotado desde los dos sentidos que le hemos atribuído: está constituído por la muerte en tanto alteridad no integrada, aún en búsqueda de sentidos y de re-presentaciones. Está constituído por los numerosos espacios-entre involucrados: objeto externo/interno; pulsión de vida/pulsión de muerte; libido de objeto/libido narcisista.

El objeto real del duelo interpela al psicoanálisis en sus límites. Esos límites están constituídos por un relacionamiento con una alteridad que toma la forma de una zona de inclusión y de pasaje de lo otro.

Todo límite en la teoría psicoanalítica y en sus modelizaciones, la pone en relación con una alteridad de manera isomórfica a la propia alteridad del inconsciente. Calificamos de paradojal un relacionamiento tal con la alteridad, que la integre preservándola al mismo tiempo como tal, y con la cuota de enigma 


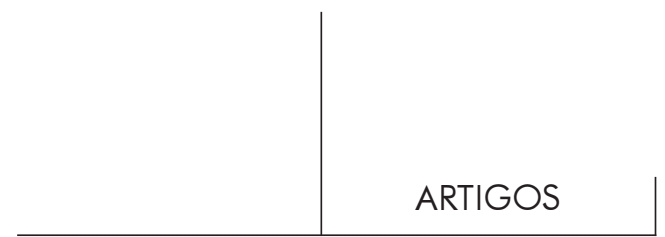

e indeterminación que le es inherente. Cuando se establece por ejemplo que la pulsión es un concepto límite, lo es a condición de que la noción misma de límite sea tomada de esta manera: menos lo que marca una delimitación clara (por ejemplo entre el territorio psicoanalítico y el biológico) que una zona-entre de différances y de intercambios no determinables.

En el caso del duelo, la realidad del objeto del duelo marca, de manera semejante, un límite a la teorización; un límite que en tanto alteridad es sujeto a un tratamiento paradojal. Objeto real y objeto psíquico se integran así en un movimiento que los complementa en su oposición, y que los mantiene en su diferencia, a través de la cual uno echa luz sobre el otro, permaneciendo al mismo tiempo indeterminado cuánto hay de uno y cuánto de otro.

Este tratamiento paradojal de los opuestos complementarios, extensivo a todas las nociones psicoanalíticas, es también el del par normal-patológico. Un abordaje psico-patológico del duelo, desde el psicoanálisis, transcurre en un espacio-entre lo normal y lo patológico, y se recentra en relación a la singularidad de un pathos y sus avatares, más que en una perspectiva normalizadora.

\section{Bibliografía}

Allouch, J. Erotique du deuil au temps de la mort sèche. Paris, E.P.E.L., 1995.

FÉDIDA, P. "Tradition tragique du psychopathologique. A propos du pathei mathos de l'Agamemnon", in Crise et contre-transfert. Paris, PUF, 1992.

"D’une psychopathologie générale à une psychopathologie fondamentale.

Note sur la notion de paradigme", in Crise et contre-transfert, op. cit.

Freud, S. "De guerra y muerte" (1915). O.C., vol. XIV. Buenos Aires, Amorrortu, 1984. "Duelo y melancolía" (1917). O.C., vol. XIV. Buenos Aires, Amorrortu, 1984.

Gantheret, F. "Per via di levare", in Incertitude d'Eros. Paris, Gallimard, 1984.

Green, A. Le travail du négatif. Paris, Minuit, 1993.

Singer, F. "Psychopathologie et paradoxalité épistémologique de la psychanalyse", tesis de Doctorado, 1998, inédita.

"Modelos en psicoanálisis", Revista Latinoamericana de Psicopatologia

Fundamental, vol. 1, no 2, jun/98, São Paulo.

\section{Resumos}

O tema da dor não foi tratado pela psicanálise na medida de sua importância psicopatológica. Existe, para ela, obstáculos epistemológicos que têm a ver com o tratamento da passagem do objeto real da dor à interioridade de seu pathos. A autora considera que uma lógica paradoxal e do negativo permitem articular essa 


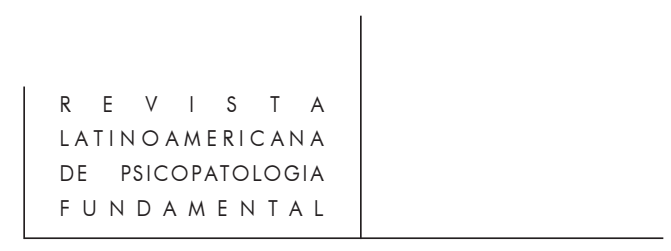

passagem: contemplar a diferença entre realidade externa-realidade interna, mas também integrar a realidade externa com a análise. O negativo está constituído, entre outros aspectos, pela complexa zona de intercâmbio entre a realidade externa da dor e a realidade interna.

O modelo da dor é um exemplo da importância metodológica de uma lógica paradoxal e do negativo que permite uma permeabilidade nos limites dos modelos, pela qual se mantêm a especificidade do modelo e a integração de uma alteridade, neste caso, a realidade do objeto da dor.

Un obstacle épistémologique en rapport avec le traitement du passage de l'objet réel du deuil à l'intériorité de son pathos a fait en sorte que le sujet du deuil n'ait pas été traité en psychanalyse de facon proportionnelle à son importance psychopathologique.

L'auteur considère qu'une logique du paradoxe et du négatif permet de contempler la différenciation entre réalité externe et interne, et d'intégrer en meme temps la réalité externe à l'analyse. Le négatif est constitué entre autres aspects, par la zone complexe d'échanges entre la réalité externe du deuil et la réalité interne.

Le modèle du deuil constitue un exemple de l'importance méthodologique d'une logique paradoxale et du négatif, qui permet une perméabilité dans les limites des modèles grace à laquelle sont préservées aussi bien la spécificité du modéle que l'intégration d'une altérité, en l'occurrence, la réalité de l'objet du deuil.

Epistemologic obstacles in relation with the way of analysing the passage between the real object of mourning and the interiority of its pathos might have contributed to insufficient development of the subject of mourning in psychoanalysis. Its development is not in accordance with its psychopathologic importance. The author thinks that a logic of paradoxality and negativity enables to take into account the differenciation between external and internal reality, and at the same time to integrate external reality into the analysis. The negativity is constituted, among other aspects, by the complexe zone of exchanges between external reality of mourning and internal reality.

The model of mourning is an example of methodologic importance of a logic of paradoxality and negativity which enables limits of models to become permeable. Thereby specificity of the model and integration of the reality of the mourning object are both taken into account. 